Pacific Journal of Mathematics

GENERALIZED RATIONAL CONVEXITY IN BANACH 


\title{
GENERALIZED RATIONAL CONVEXITY IN BANACH ALGEBRAS
}

\author{
Gustavo Corach and Fernando D. Suárez
}

\begin{abstract}
Let $A$ be a complex commutative Banach algebra with identity and let $F$ be a closed subset of its spectrum $X(A)$. There are several hulls, associated with $F$, which are useful in the study of approximation, interpolation and separation problems: the polynomially and rationally convex hulls are the most popular, the $A$-convex hull has also been considered and there are also holomorphically convex hulls. In this paper we introduce a family of hulls, denoted by $R_{n}(F)$, and we study some relations between these hulls and several known objects and invariants in commutative Banach algebras.
\end{abstract}

We study the relations between $R_{n}(F)$ and the generalized Shilov boundaries introduced by Basener [1] and Sibony [17], the topological stable rank, introduced by Rieffel [15], the dimension of $X(A)$, the minimal number of generators of $A$, etc. We describe briefly the contents of the paper. In $\S 1$ we introduce the hulls $R_{n}(F)$ and prove several basic properties of them. In $\S 2$ we consider those $F$ 's such that $R_{n}(F)=X(A)$; the intersection of all those $F$ 's, denoted by $\Gamma_{n}(A)$, seems to play a role similar to that of the generalized boundaries $S_{n}(A)$ of Basener and Sibony; we prove that $\Gamma_{0}(A)=S_{0}(A) \subset$ $\Gamma_{1}(A) \subset S_{1}(A) \cdots$ but, in general, $\Gamma_{n}(A)$ is strictly contained in $S_{n}(A)$. In $\S 3$ we introduce the invariant $r(A)=\min \left\{n \geq 0: R_{n}(F)=F\right.$ for every $F$, which we call the rationality of $A$, and we relate it with the sets $\Gamma_{n}(A)$ and $S_{n}(A)$. In $\S 4$ we study the relationship between the topological stable rank of $A$ with the new notions. In $\S 5$ we prove that $d(A) \leq r(A)+\gamma(A) \leq 2 \gamma(A)$ where $d(A)$ is the (covering) dimension of $X(A)$ and $\gamma(A)$ is the minimal number of generators of $A$; the proof of this result uses known facts on Čech cohomology groups of some compact subsets of $\mathbb{C}^{n}$, due to Andreotti and Narasimhan and Duchamp and Stout (see [7]).

Section 6 contains a generalization of a result of Forelli [11] and $\S 7$ contains some results about the hulls $R_{n}$ in the particular case of the algebra $H^{\infty}$. Finally, in $\S 8$ we collect several open problems. There are several direct predecessors of this paper: one is a work of Csordas and Reiter [5], who introduced the hulls $R_{1}$ (which they call $L$-sets) and the 
condition $r(A)=1$ (this is a separating algebra in their terminology); another ancestor is a paper of Eifler [9], who studied $F$ 's such that $R_{1}(F)=X(A)$ (these are his inverting sets for $A$ ); finally we have taken several facts about hulls from Stout's book [19]. We thank the referee for his valuable comments, in particular for correcting a mistake in Theorem 7.1.

Preliminaries. In this paper a Banach algebra $A$ means a complex commutative Banach algebra with identity. The spectrum of $A$ is the set $X(A)$ of all (identity preserving) homomorphisms $A \rightarrow \mathbb{C}$ (called characters). Every character is continuous; so we can consider on $X(A)$ the weak * topology of the dual $A^{*}$. With this topology $X(A)$ is a compact Hausdorff space. The Gelfand map of $A$ is the homomorphism $g: A \rightarrow C(X(A))$ defined by $g(a)=\widehat{a}, \widehat{a}(h)=h(a)(h \in X(A))$. The letter $F$ denotes a non-void closed subset of $X(A)$ and $A_{F}$ is the closed subalgebra of $C(F)$ generated by the restrictions $\widehat{a} \mid F(a \in A)$. In general, for a space $X, C(X)$ is the algebra of all complex continuous functions on $X$. For $a \in A,\|\widehat{a}\|_{F}=\|\widehat{a} \mid F\|_{\infty}$. Given $a \in A^{n}$ we set $\sigma(A)=\{h(z): h \in X(A)\}=\widehat{a}(X(A))$. The zero set of $a \in A^{n}$ is $Z_{a}=\{h \in X(A): h(a)=0\}$. It is known that $X\left(A_{z_{a}}\right)$ is homeomorphic to $Z_{a}$. If $A$ is a Banach algebra and $n \geq 1$ we use in $A^{n}$ the norm $\|a\|=\left\|\left(a_{1}, \ldots, a_{n}\right)\right\|=\left(\sum_{k=1}^{n}\left\|a_{i}\right\|^{2}\right)^{1 / 2}$. This holds, in particular, for $z \in C^{n}$, where we write $|z|$ instead of $\|z\|$. In $\S 6$ we denote by $H^{k}$ the $k$ th Čech cohomology functor with integer coefficients.

\section{Generalized rational hulls.}

1.1. Definitions. Given a closed subset $F$ of $X(A)$ define $R_{0}(F)=$ $\left\{h \in X(A):|h(a)| \leq\|\widehat{a}\|_{F}(a \in A)\right\}$. For $n \geq 1$ and $a \in A^{n}$ we put $\Delta(F, a)=\widehat{a}^{-1}(\widehat{a}(F))=\{h \in X(A): h(a) \in \widehat{a}(F)\}$ and $R_{n}(F)=$ $\bigcap\left\{\Delta(F, a): a \in A^{n}\right\}$.

1.2. Remarks. (i) $R_{0}(F)$ is usually denoted by $\widehat{F}$ and referred to as the $A$-convex hull of $F$

(ii) The sets $R_{1}(F)$ were first considered in [5]; see also [19, $\mathrm{p}$. 369].

(iii) Given a compact subset $K$ of $\mathbb{C}^{\Lambda}$ (where $\Lambda$ is an arbitrary set and $\mathbb{C}^{\Lambda}$ is provided with the product topology) and a set of functions 
$\mathscr{S}$ defined on $\mathbb{C}^{\Lambda}$ we can define the hulls

$$
\begin{aligned}
& R_{0}(K, \mathscr{S})=\left\{z \in \mathbb{C}^{\Lambda}:|f(z)| \leq\|f\|_{K} \text { for all } f \in \mathscr{S}\right\}, \\
& R_{n}(K, \mathscr{S})=\left\{z \in \mathbb{C}^{\Lambda}: f(z) \in f(K) \text { for all } f=\left(f_{1}, \ldots, f_{n}\right) \in \mathscr{S}^{n}\right\} \\
& \qquad(n \geq 1) .
\end{aligned}
$$

If $\mathscr{S}=\mathscr{P}$ is the algebra of all complex polynomials in the variables $X_{\lambda}(\lambda \in \Lambda), R_{0}(K, \mathscr{P})$ is the usual polynomial hull of $K$ (see [19]). If $\mathscr{S}=\mathscr{R}_{K}$ the algebra of all rational functions with poles off $K, R_{0}\left(K, \mathscr{P}_{K}\right)$ is the rational hull of $K$. It is well known that $R_{0}\left(K, \mathscr{R}_{K}\right)=R_{1}(K, \mathscr{P})$. These classical hulls motivate the name of generalized rational hulls given to the sets $R_{n}(F)$, which should be denoted by $R_{n}(F, \widehat{A})$.

We collect some facts about $R_{n}(F)$ in the next proposition.

1.3. Proposition. (i) $X(A) \supset \cdots \supset R_{n}(F) \supset R_{n+1}(R) \supset \cdots \supset$ $F(n \leq 0)$.

(ii) If $R_{n}(F)=F$ then $R_{k}(F)=F$ for all $k \geq n$.

(iii) If $R_{n}(F)=X(A)$ then $R_{k}(F)=X(A)$ for all $k \leq n$.

(iv) For $h \in X(A), h \notin f R_{0}(F)$ if and only if for every $\varepsilon>0$ there exists $a \in A$ such that $h(a)=1$ and $\|\widehat{a}\|_{F}<\varepsilon$.

(v) If $n \geq 1$ and $h \in X(A)$, then $h \notin R_{n}(F)$ if and only if there exists $a \in A^{n}$ such that $h(a)=0$ and $0 \notin \widehat{a}(F)$.

(vi) If $n \geq 1$ and $D$ is a dense subset of $A^{n}$ then

$$
R_{n}(F)=\bigcap\{\Delta(a, F): a \in D\} .
$$

(vii) For every $k \geq 0$ it holds

$$
\bigcap\left\{R_{n}(F): n \geq 0\right\}=\bigcap\left\{R_{n}(F): n \geq k\right\}=F .
$$

Proof. (i) Obviously $\Delta(F, a) \supset F$ for every $a \in A^{n}$, so $R_{n}(F) \supset F$ for $n \geq 1$. For $a \in A^{n}, \Delta(F, a)=\Delta(F,(a, 0))$, so $R_{n+1}(F) \subset R_{n}(F)$ for $n \geq 1$. The case $R_{1}(F) \subset R_{0}(F)$ is clear.

(ii) and (iii) follow easily from (i).

(iv) If $h \in X(A) \backslash R_{0}(F)$, there is $a \in A$ such that $|h(a)|>\|\hat{a}\|_{F}$. Multiplying $a$ by an appropriate constant we can suppose that $1=$ $h(a)>\|\widehat{a}\|_{F}$. Finally, replacing $a$ by $a^{n}$ with $n$ large enough we get $h(a)=1$ and $\|\widehat{a}\|_{F}<\varepsilon$. The converse is obvious.

(v) If $h \in X(A) \backslash R_{n}(F)$, there is $a \in A^{n}$ such that $h(a) \notin \widehat{a}(F)$. If $b=a-h(a)$, then $h(b)=0$ and $0 \notin \widehat{b}(F)$. 
(vi) It suffices to prove that $R_{n}(F) \supset \bigcap\{\Delta(F, a): a \in D\}$. Let $h \in$ $X(A) \backslash R_{n}(F)$. By (v) there is $a \in A^{n}$ such that $h(a)=0 \notin \widehat{a}(F)$. Then $\delta=\inf \{|k(a)|: k \in F\}>0$ and we can choose $b \in D$ such that $\|a-b\|<\delta / 2$. We shall show that $|h(b)|<\inf \{|k(b)|: k \in F\}$, which will imply that $h(b) \notin \widehat{b}(F)$, i.e. that $h \notin \bigcap\{\Delta(F, d): d \in D\}$. Now, $\|a-b\|<\delta / 2$ implies that $|h(b)|=|h(b)-h(a)| \leq\|a-b\|<\delta / 2$, so it suffices to prove that $|k(b)| \geq \delta / 2$ for every $k \in F$. If $k \in F$ then ||$k(a)|-| k(b)|| \leq|k(a)-k(b)| \leq\|a-b\|<\delta / 2$ so we get $|k(b)| \geq|k(a)|-\delta / 2 \geq \delta-\delta / 2=\delta / 2$.

(vii) Part (i) implies that, for each $k \geq 0, \bigcap\left\{R_{n}(F): n \geq k\right\}=$ $\bigcap\left\{R_{n}(F): n \geq 0\right\} \supset F$. If $h \notin F$, for every $k \in F$ there is $a_{k} \in A$ such that $\widehat{a}_{k}(k) \neq 0$ and $\widehat{a}_{k}(h)=0$.

Let $U_{k}$ be an open neighborhood of $k$ such that $\widehat{a}_{k}(l) \neq 0$ for all $l \in U_{k}$. By compactness, there are $k_{1}, \ldots, k_{n} \in F$ such that $F \subset$ $U_{k_{1}} \cup \cdots \cup U_{k_{n}}$. If $a=\left(a_{k_{1}}, \ldots, a_{k_{n}}\right)$ then $h(a)=0$ and $0 \notin \widehat{a}(F)$. This means, by $(\mathrm{v})$, that $h \notin R_{n}(F)$, so $h \notin \bigcap R_{n}(F)$

1.4. Remarks. (i) The equality $\bigcap R_{n}(F)=F$ can also be proved by means of the axiomatic joint spectra theory of Zelazko [25] (see also Curto [6], Eschmeier [10] and Vasilescu [23]). A spectral system on a Banach algebra $A$ is a rule $\widetilde{\sigma}$ which assigns to each $a \in A^{n}(n \geq 1)$ a compact subset $\tilde{\sigma}(a)$ of $\mathbb{C}^{n}$ such that $\tilde{\sigma}(a) \subset \sigma(a), p_{1}(\tilde{\sigma}(a, b))=\tilde{\sigma}(a)$ and $p_{2}(\tilde{\sigma}(a, b))=\tilde{\sigma}(b)$, where $p_{1}: \mathbb{C}^{n} \times \mathbb{C}^{m} \rightarrow \mathbb{C}^{n}, p_{2}: \mathbb{C}^{n} \times \mathbb{C}^{m} \rightarrow$ $\mathbb{C}^{m}$ are the usual projections. Given a spectral system $\tilde{\sigma}$ on $A, \Delta=$ $\bigcap\left\{\widehat{a}^{-1}(\widetilde{\sigma}(a)): a \in A^{n}, n \geq 1\right\}$ is the only closed subset of $X(A)$ such that $\tilde{\sigma}(a)=\widehat{a}(\Delta)$ (see [10,1.1], for instance). It is easily seen that, for every $F, \tilde{\sigma}: a \rightarrow \widehat{a}(F)$ is a spectral system with $\Delta=\bigcap\left\{R_{n}(F): n \geq 1\right\}$ and $\tilde{\sigma}(a)=\widehat{a}(F)$ by definition. By the uniqueness of $\Delta$ it follows that $\Delta=F$.

(ii) Observe that each $R_{n}$ is an involution operator in the sense that $R_{n}\left(F_{n}(F)\right)=R_{n}(F)$.

\section{The condition $R_{n}(F)=X(A)$.}

2.1. Proposition. The following conditions are equivalent.

(1) $R_{n}(F)=X(A)$.

(2) For every $a \in A^{n}, \sigma(a)=\widehat{a}(F)$.

(3) For every $a \in A^{n}, Z_{a} \cap F=\varnothing$ implies that $Z_{a}=\varnothing$.

Proof. (1) $\Rightarrow(2)$. Suppose that $\sigma(a) \neq \widehat{a}(F)$ for some $a \in A^{n}$. Then there is $h \in X(A)$ such that $h(a) \notin \widehat{a}(F)$. This means that $h \notin \Delta(F, a)$ 
so $h \notin R_{n}(F)$, which contradicts (1). (2) $\Rightarrow$ (3). Take $a \in A^{n}$ such that $Z_{a} \neq \varnothing$. Then $0 \in \sigma(a)=\widehat{a}(F)$ and there exists $k \in F$ such that $k(a)=0$, or, which is the same, $k \in Z_{a} \cap F$.

(3) $\Rightarrow(1)$. If $h \notin R_{n}(F)$, there exists $a \in A^{n}$ such that $h(a)=0$ and $0 \notin \widehat{a}(F)$; in other terms, $h \in Z_{a}$ and $Z_{a} \cap F=\varnothing$; this contradicts (3).

2.2. Definition. Let $n \geq 1$. $F$ is $n$-inverting (for $A$ ) if $0 \notin \widehat{a}(F) \Rightarrow$ $a \in U_{n}(A)=\left\{c \in A^{n}: \sum_{k=1}^{n} A c_{k}=A\right\}$, for all $a \in A^{n}$. For $n=1$ this is a notion due to Eifler [9].

It is easy to see that $a \in U_{n}(A)$ if and only if $0 \notin \sigma(a)$. Thus, $F$ is $n$-inverting if and only if $R_{n}(F)=X(A)$. We say that $F$ is 0 -inverting if $R_{0}(F)=X(A)$.

The intersection of all $n$-inverting subsets of $X(A)$ will be denoted $\Gamma_{n}(A)$ :

$$
\Gamma_{n}(A)=\bigcap\left\{F: R_{n}(F)=X(A)\right\} \quad(n \geq 0) .
$$

The proof of the next result follows easily from 1.3 (iii) and the definition of the Shilov boundary (see also the next paragraph):

2.3. Proposition. (i) $\Gamma_{0}(A)$ is the Shilov boundary of $A$.

(ii) $\Gamma_{0}(A) \subset \Gamma_{1}(A) \subset \Gamma_{2}(A) \subset \cdots$.

Therefore $\Gamma_{n}(A)$ is non-void for all $n \geq 0$.

The next result relates the notion of hulls $R_{n}$ to that of generalized Shilov boundaries introduced by Basener [1] and Sibony [17]. Recall the definitions. A boundary for $A$ is a closed subset $F$ of $X(A)$ such that $\|\widehat{a}\|_{F}=\|\widehat{a}\|_{X(A)}(a \in A)$. The Shilov boundary $S_{0}(A)$ is the intersection of all boundaries and it turns to be itself a boundary. For $n \geq 1$, the $n$th boundary of $A$ is the closure in $X(A)$ of $\bigcup\{S)_{0}\left(A_{Z_{a}}\right): a \in$ $\left.A^{n}\right\}$ (this makes sense because $S_{0}\left(A_{Z_{a}}\right) \subset X\left(A_{Z_{a}}\right)=Z_{a} \subset X(A)$ ). We denote it by $S_{n}(A)$.

An interesting result of Tonev [21] is the following: $S_{n}(A)$ is the intersection of all $n$-boundaries of $A$ and it is itself an $n$-boundary, where a closed subset $E$ of $X(A)$ is an $n$-boundary (for $A$ ) if $\min \{|k(a)|: k \in E\}=\min \{|k(a)|: k \in X(A)\}$ for all $a \in U_{n+1}(A)$.

For more information on these generalized boundaries the reader is referred to $[1,17,21]$.

\subsection{Proposition. For $n \geq 0 R_{n}\left(S_{n}(A)\right)=X(A)$.}

Proof. The case $n=0$ is trivial. For $n \geq 1$ suppose, on the contrary, that there is $h \in X(A) \backslash R_{n}\left(S_{n}(A)\right)$. Then, by $1.3(\mathrm{v})$, there exists $a \in A^{n}$ 
such that $h(a)=0$ and $0 \notin \widehat{a}\left(S_{n}(A)\right)$. This means that $S_{n}(A) \cap Z_{a}=\varnothing$. Now $Z_{a} \neq \varnothing$ and $S_{0}\left(A_{Z_{a}}\right) \neq \varnothing$ but, by definition of $S_{n}(A), S_{0}\left(A_{Z_{a}}\right) \subset$ $S_{n}(A) \cap Z_{a}$, contradiction. Thus $R_{n}\left(S_{n}(A)\right)=X(A)$, as claimed.

\subsection{Corollary. $\Gamma_{n}(A) \subset S_{n}(A)(n \geq 1)$.}

\subsection{Proposition. For every $n \geq 1 \Gamma_{n}(A) \supset S_{n-1}(A)$.}

Proof. Suppose, on the contrary, that there exist $h \in S_{n-1}(A)$ and a closed subset $F$ of $X(A)$ not containing $h$ such that $R_{n}(F)=X(A)$. Consider an open neighborhood $U$ of $h$ which does not meet $F$. From Tonev's characterization of $S_{n-1}(A)$ [21] there is $a \in U_{n}(A)$ such that

$$
\begin{aligned}
\min & \{|k(a)|: k \in X(A)\}=\min \{|k(a)|: k \in k \in U\} \\
& <\min \{|k(a)|: k \in X(A) \backslash U\} \leq \min \{|k(a)|: k \in F\} .
\end{aligned}
$$

Let $h_{0} \in U$ such that $\left|h_{0}(a)\right|=\min \{|k(a)|: k \in U\}$. Then $\left|h_{0}(a)\right|<$ $\min \{|k(a)|: k \in F\}$, by the inequalities above, so that $h_{0}(a) \notin \widehat{a}(F)$, which means that $h_{0} \notin R_{n}(F)$, contradiction.

2.7. Corollary. $\Gamma_{0}(A)=S_{0}(A) \subset \Gamma_{1}(A) \subset S_{1}(A) \subset \cdots$. Therefore $\bigcup_{n \geq 0}^{\infty} S_{n}(a)=\bigcup_{n \geq 0} \Gamma_{n}(A)$.

We prove now that the unions considered at 2.7 are dense in $X(A)$.

2.8. Proposition. $\bigcup\left\{S_{n}(A): n \geq 0\right\}$ is dense in $X(A)$.

Proof. Suppose on the contrary that there exists $h \in X(A) \backslash F$, where $F$ is the closure of $\bigcup S_{n}(A)$.

Let $U=X(A) \backslash F$. By $1.3(\mathrm{v})$ there exist $n \in \mathbb{N}$ and $a \in A^{n}$ such that $h(a)=0$ and $\widehat{a}(F) \not \supset 0$. Then $F \cap Z_{a}=\varnothing$ so $S_{0}\left(A_{Z_{a}}\right) \subset Z_{a} \subset U$ and $S_{n}(A) \cap U$ is non-void which is absurd by the definition of $U$. Thus $F=X(A)$ as claimed.

2.9. REMARK. It is worth noting that, in general, $\Gamma_{n}(A)$ is not an $n$-inverting set. For this, let $A=A\left(\mathbb{D}^{n}\right)=$ the $n$-polydisc algebra, $\alpha=\left(\alpha_{1}, \ldots, \alpha_{n}\right) \in \mathbb{C}^{n}$ with $\left|\alpha_{k}\right|<1(k=1, \ldots, n)$ and $\varphi_{\alpha}^{(k)} \in A$ defined by $\varphi_{\alpha}^{(k)}(z)=\left(z-\alpha_{k}\right) /\left(1-z \bar{\alpha}_{k}\right)$. By a result of Rudin [16], Theorem 4.7.2 and the definition of $R_{k}, R_{1}\left(K_{\alpha} \cup \mathbb{T}^{n}\right)=\mathbb{D}^{n}=X(A)$, where $K_{\alpha}$ is the image of $\varphi_{\alpha}=\left(\varphi_{\alpha}^{(1)}, \ldots, \varphi_{\alpha}^{(n)}\right)$ and $\mathbb{T}=\{z \in \mathbb{D}:|z|=1\}$. Then, by its definition, $\Gamma_{1}(A)$ is contained in $\bigcap\left(K_{\alpha} \cup \pi^{n}\right)$ where the intersection is taken over all $\alpha \in \mathbb{C}^{n}$ with $\left|\alpha_{k}\right|<1$ for all $k$, which is obviously $\mathbb{T}^{n}$. Moreover, by $2.3, \Gamma_{1}(A) \supset \Gamma_{0}(A)=S_{0}(A)=\mathbb{T}^{n}$ so that $\Gamma_{1}(A)=\mathbb{T}^{n}$. 
However $R_{1}\left(\mathbb{T}^{n}\right)=\mathbb{T}^{n}$, for if $w \in \mathbb{D}^{n} \backslash \mathbb{T}^{n}$ then $\left|w_{k}\right|<1$ for some $k=1, \ldots, n$ and the polynomial $p\left(z_{1}, \ldots, z_{n}\right)=z_{i}-w_{i}$ vanishes at $w$ and has no zero at $\mathbb{T}^{n}$.

3. The condition $R_{n}(F)=F$. Let $r(A)=\min \left\{n \geq 0: R_{n}(F)=\right.$ $F \forall F\}$ and $r(A)=+\infty$ if there is no such $n$; we call $r(A)$ the rationality of $A$.

3.1. Proposition. (i) $R_{0}(F)=F$ if and only if for every $\varepsilon>0$ and $h \in X(A) \backslash F$ there exists $a \in A$ such that $h(a)=1$ and $\|\widehat{a}\|_{F}<\varepsilon$.

(ii) If $A$ is regular then $r(A)=0$.

(iii) Suppose that there exists $a \in A^{n}$ such that $\widehat{a}: X(A) \rightarrow \mathbb{C}^{n}$ is injective. Then $r(A) \leq n$.

Proof. (i) follows easily from 1.3 (iv).

(ii) If $A$ is regular and $h \in X(A) \backslash F$ there exists $a \in A$ such that $\widehat{a} \mid F=0$ and $h(a)=1$; apply (i).

(iii) If $\widehat{a}$ is injective, for every $F$ it holds $\Delta(a, F)=\widehat{a}^{-1}(\widehat{a}(F))=F$, so $R_{n}(F)=\bigcap \Delta(a, F)$ and $r(A) \leq n$.

3.2. Remarks. (i) In [24] Wilken says that $A$ is approximately regular (on $X(A)$ ) if for each $h \in X(A)$, each closed set $F$ in $X(A)$ not containing $h$ and each $\varepsilon>0$ there is $a \in A$ with $h(a)=1$ and $\|\widehat{a}\|_{F}<\varepsilon$. By 3.1 (i) $A$ is approximately regular if and only if $r(A)=0$.

(ii) Let $A$ be $n$-generated, in the sense that there are $a_{1}, \ldots, a_{n}$ in $A$ such that the subalgebra generated by $a_{1}, \ldots, a_{n}$ is dense in $A$. Then 3.1 (iii) implies that $r(A) \leq n$, because if $a=\left(a_{1}, \ldots, a_{n}\right)$ then $\widehat{a}: X(A) \rightarrow$ $\sigma(a) \subset \mathbb{C}^{n}$ is a homeomorphism. Thus, $r(A) \leq \gamma(A)$ if $\gamma(A)$ is the minimum number of generators of $A$. However, this inequality is not sharp. In fact, if $X$ is an infinite dimensional compact space, e.g. $X=[0,1]^{\mathrm{N}}$, then $A=C(X)$ is regular, so $r(A)=0$ by 3.1 (ii), and $\gamma(A)=+\infty$. We will see later a more precise relationship between $r(A), \gamma(A)$ and the dimension of $X(A)$.

(iii) Suppose that there exist $a_{1}, \ldots, a_{n}$ in $A$ such that the closed full subalgebra generated by them is $A$. Then $\widehat{a}$ is injective and $r(A) \leq n$.

(iv) The condition $r(A) \leq 1$ has been introduced by Csordas and Reiter [5], who called such $A$ 's "separating algebras". Most of their results can be generalized to our setting.

3.3. Proposition. If $r(A) \leq n$ then $\Gamma_{n}(A)=S_{n}(A)=X(A)$. 
Proof. By 2.5 it suffices to see that $\Gamma_{n}(A)=X(A)$. But if $r(A) \leq$ $n$ the only closed set $F$ in $X(A)$ which satisfies $R_{n}(F)=X(A)$ is $X(A)$.

3.4. ExAmples. Given a compact subset $X$ of $\mathbb{C}^{n}$ let $A(X)$ be the algebra of all maps $X \rightarrow \mathbb{C}$ which are holomorphic in the interior of $X$. Then $r(A(X)) \leq n$ if $X$ is polynomially convex, for in this case the coordinate functions $z_{1}, \ldots, z_{n}$ generate $A(X)$. In particular, if $\mathbb{D}$ is the closed unit disc $r(A(\mathbb{D}))=1$ because $R_{0}(F) \neq F$ for every $F$ with $\mathbb{C} \backslash F$ disconnected, so $0<r(A(\mathbb{D})) \leq 1$. In general, $r\left(A\left(\mathbb{D}^{n}\right)\right)=n$. We will return later to this example.

3.5. Proposition. If $R_{n}(F)=F$ then $F$ is the intersection of a family of sets $P_{a, \delta}=\{h \in X(A):|h(a)|>\delta\}$ for some elements $a \in A^{n}$ and $\delta>0$.

Proof. Let $h_{0} \in X(A) \backslash F$. By 1.3 (v) there is $a \in A^{n}$ such that $h_{0}(a)=0 \notin \widehat{a}(F)$. Let $\delta=\min \{|h(a)|: h \in F\}$. Then $\delta>0$ and $h_{0} \notin P_{a, \delta / 2}$. Thus, $F=\bigcap P_{a, \delta}$ for a family $\{(a, \delta)\}$ in $A^{n} \times \mathbb{R}^{+}$.

4. The topological stable rank, $R_{n}$ and $S_{n}$. The topological stable rank of $A$ is the least $n$ such that $U_{n}(A)$ is dense in $A^{n}$; we denote it by $\operatorname{tsr}(A)$. This is a notion introduced by Rieffel [15] which replaced efficiently, in the case of Banach algebras, the more algebraic stable range conditions of Hyman Bass [2].

4.1. Proposition. Suppose that $\operatorname{tsr}(A) \leq n$. Then $R_{n}\left(S_{n-1}(A)\right)=$ $X(A)$.

Proof. By $[4,1.10]$ there is a dense subset $D$ of $A^{n}$ such that $\sigma(a)$ has no interior points, for every $a \in D$. Then $\sigma(a)$ coincides with its boundary $\partial \sigma(a)$. But, by a result of Tonev [21] $\partial \sigma(a) \subset \widehat{a}\left(S_{n-1}(A)\right)$ $\left(a \in A^{n}\right)$. Thus, for every $a \in D, \sigma(a)=\widehat{a}\left(S_{n-1}(A)\right)$, so that by 1.3 (vi),

$$
\begin{aligned}
R_{n}\left(S_{n-1}(A)\right) & =\bigcap\left\{\Delta\left(a, S_{n-1}(A)\right): a \in D\right\} \\
& =\bigcap\left\{\hat{a}^{-1}(\sigma(a)): a \in D\right\}=X(A),
\end{aligned}
$$

because $\widehat{a}^{-1}(\sigma(a))=X(A)\left(a \in A^{n}\right)$.

4.2. Corollary. Suppose that $\operatorname{tsr}(A) \leq n$ and $r(A) \leq n$. Then $S_{n-1}(A)=X(A)$. 
4.3. Proposition. Suppose that $\operatorname{tsr}(A)=1$. Then $R_{0}(F)=R_{1}(F)$.

Proof. It always holds $R_{0}(F) \supset R_{1}(F),(1.3(\mathrm{i}))$. Let $h \notin R_{1}(F)$. By 1.3 (v) there is $a \in A$ such that $h(a)=0$ and $\min \{|k(a)|: k \in F\}>1$. By hypothesis, the invertible elements form a dense subset of $A$, so there is an invertible $u \in A$ with $\|a-u\|<1 / 2$. Then $|h(u)|<1 / 2$ and $\min \{|k(u)|: k \in F\}>1 / 2$, so $\left|h\left(u^{-1}\right)\right|>2$ and $\left\|u^{-1}\right\|_{F}<2$, which implies that $h \notin R_{0}(F)$.

4.4. Corollary. Suppose that $\operatorname{tsr}(A)=1$ and $r(A) \leq 1$. Then $r(A)=0$.

It is an open problem if $\operatorname{tsr}(A)$ and $\operatorname{tsr}(C(X(A)))$ coincide. We present two results relating this problem with the invariant $r(A)$.

4.5. Proposition. Suppose that $r(A)=0$. Then $\operatorname{tsr}(C(X(A))) \leq$ $\operatorname{tsr}(A)$.

Proof. Suppose that $\operatorname{tsr}(C(X(A))) \geq n+1$. Then there exist $F$, a closed subset of $X(A)$, and $\varphi_{1}, \ldots, \varphi_{n} \in C(X(A))$ such that $\varphi(F)=$ $\left(\varphi_{1}(F), \ldots, \varphi_{n}(F)\right) \subset \mathbb{C}_{*}^{n} \backslash\{0\}$ and $\varphi \mid F$ admits no extension to $C\left(X(A), \mathbb{C}_{*}^{n}\right)$ [22]. By the fact that $R_{0}(F)=F$, there is $\theta \in A_{F}^{n}$ lying in the component of $\varphi \mid F$ in $C\left(F, C_{*}^{n}\right)$ : in fact, $X\left(A_{F}\right)=R_{0}(F)=F$ and it is well known that, for a Banach algebra $B, U_{n}(B)$ and $C\left(X(B), \mathbb{C}_{*}^{n}\right)$ are homotopy equivalent [20]. Now, by definition of $A_{F}, \widehat{A} \mid F$ is dense in $A_{F}$ so that there is $a \in A^{n}$ such that $\widehat{a} \mid F$ belongs to the component of $\theta$ in $C\left(F, \mathbb{C}_{*}^{n}\right)$. In particular, $\widehat{a} \mid F$ admits no extension to $C\left(X(A), \mathbb{C}_{*}^{n}\right)$. By the results of Vaserstein [22] or Rieffel [15], this means precisely that $\widehat{a}$ does not belong to the closure of $U_{n}\left(C(X(A))\right.$ ) (in $\left.C(X(A))^{n}\right)$, which implies that $a$ does not belong to the closure of $U_{n}(A)$ (in $A^{n}$ ). Thus $\operatorname{tsr}(A) \geq n+1$. This proves that $\operatorname{tsr}(A) \geq \operatorname{tsr}(C(X(A)))$.

4.6. Corollary. If $r(A) \leq 1$ and $\operatorname{tsr}(A)=1$ then $\operatorname{tsr}(C(X(A)))=1$.

Proof. Combine 4.5 and 4.4.

4.7 Problem. It is not known if $\operatorname{tsr}(A)=\operatorname{tsr}(C(X(A))$, in general, even for regular algebras.

5. The main result. This section contains the main result of the paper, which states that the topological dimension of $X(A)$ is at most $\gamma(A)+r(A)$. We need some results about the cohomology of certain compact subsets of $\mathbb{C}^{n}$. 
5.1. LeMMA. Let $R_{1}, \ldots, R_{m}$ be rational convex compact subsets of $\mathbb{C}^{n}$ and $J=\bigcup_{k=1}^{m} R_{k}$. Then $H^{n+i}(J)=0$ for all $i \geq m$.

Proof. We proceed by induction in $m$. Let $m=1$. If $K \subset \mathbb{C}^{n}$ is polynomially convex then $H^{n+i}(K)=0$ for all $i \geq 0$ [7]. If $K \subset \mathbb{C}^{n}$ is rationally convex, then $K$ is homeomorphic to some polynomially convex $K^{\prime} \subset \mathbb{C}^{n+1}\left[19\right.$, p. 373] so that $H^{n+i}(K)=H^{n+i}\left(K^{\prime}\right)=0$ for all $i \geq 1$. Suppose that the assertion holds for $1,2, \ldots, m$ and let $J=\bigcup_{1}^{m+1} R_{k}$, where each $R_{k}$ is rationally convex, $R_{k} \subset \mathbb{C}^{n}$. Consider the Mayer-Vietoris exact sequence

$$
\begin{aligned}
\cdots & \rightarrow H^{n+i-1}\left(\left(\bigcup_{1}^{m} R_{k}\right) \cap R_{m+1}\right) \rightarrow H^{n+i}\left(\bigcup_{1}^{m+1} R_{k}\right) \\
& \rightarrow H^{n+i}\left(\bigcup_{1}^{m} R_{k}\right) \oplus H^{n+i}\left(R_{m+1}\right) \rightarrow \cdots .
\end{aligned}
$$

Then, by inductive hypothesis, if $i \geq m+1$,

$$
H^{n+i}\left(\bigcup_{1}^{m} R_{k}\right)=0, \quad H^{n+i}\left(R_{m+1}\right)=0
$$

and

$$
H^{n+i-1}\left(\left(\bigcup_{1}^{m} R_{k}\right) \cap R_{m+1}\right)=H^{n+i-1}\left(\bigcup_{1}^{m} R_{k} \cap R_{m+1}\right)=0
$$

because each $R_{k} \cap R_{m+1}$ is rationally convex. Then, by the exactness, $H^{n+i}(J)=H^{n+i}\left(\bigcup_{1}^{m+1} R_{k}\right)=0$.

We say that a compact subset $K$ of $\mathbb{C}^{n}$ is $k$-rationally convex if $R_{k}(K)=K$, where

$$
R_{k}(K)=\bigcap\left\{p^{-1}(p(K)): p=\left(p_{1}, \ldots, p_{k}\right), p_{i} \in \mathbb{C}\left[t_{1}, \ldots, t_{n}\right]\right\} .
$$

This set is denoted $h_{k}^{r}(K)$ in Słodkowski's paper [18, p. 323].

5.2. Corollary. Let $K$ be an $(n-1)$-rationally convex subset of $\mathbb{C}^{n}$. Then $K$ does not separate $\mathbb{C}^{n}$ (i.e., $\mathbb{C}^{n} \backslash K$ is connected).

Proof. Suppose, on the contrary, that $\mathbb{C}^{n} \backslash K$ is disconnected, and assume that $K \subset B=\left\{z \in C^{n}:|z|=\left(\sum_{i=1}^{n}\left|z_{i}\right|^{2}\right)^{1 / 2} \leq M\right\}$. Then there are polynomials $p_{1}, \ldots, p_{n-1}$ such that $p\left(z_{0}\right)=0 \notin p(K)$, where $p=\left(p_{1}, \ldots, p_{n-1}\right)$. Then there is $\delta>0$ such that $K \subset \bigcup_{k=1}^{n-1} R_{k}$, where $R_{k}=\left\{z \in B:\left|p_{k}(z)\right| \geq \delta\right\}$. Observe that each $R_{k}$ is rationally convex, 
so that, by $6.1, H^{n+i}\left(\bigcup_{k=1}^{n-1} R_{k}\right)=0$ for all $i \geq n-1$; in particular $H^{2 n-1}\left(\bigcup_{k=1}^{n-1} R_{k}\right)=0$, so that $\bigcup_{k=1}^{n-1} R_{k}$ does not separate $\mathbb{C}^{n}[\mathbf{1 4}, \mathrm{p}$. 100]. But $z_{0} \in B \backslash \bigcup_{k=1}^{n-1} R_{k}, K \subset \bigcup_{k=1}^{n-1} R_{k}$ and $K$ separates $\mathbb{C}^{n}$, so $\bigcup_{k=1}^{n-1} R_{k}$ should separate $\mathbb{C}^{n}$, contradiction.

5.3. Corollary. For every compact subset $K$ of $\mathbb{C}^{n}, R_{n-1}(K)$ contains all bounded components of $\mathbb{C}^{n} \backslash K$.

5.4. Remark. It is false, in general, that $R_{n-1}(K)$ consists, exactly, of $K$ and all bounded components of $\mathbb{C}^{n} \backslash K$. For instance, at 2.9 with $n=2$, any $\alpha=\left(\alpha_{1}, \alpha_{2}\right)$ and $K=K_{\alpha} \cup \mathbb{T}^{2}$ it holds $R_{1}(K)=\mathbb{D}^{2}$ and $K$ does not separate $\mathbb{C}^{2}$.

5.5. Proposition. If $A$ is generated by $a_{1}, \ldots, a_{n}$ then $R_{k}(\widehat{a}(F))=$ $\widehat{a}\left(R_{k}(F)\right)$ for every closed subset $F$ of $X(A)$.

Proof. The inclusion $\widehat{a}\left(F_{k}(F)\right) \subset R_{k}(\widehat{a}(F))$ holds in general: if $h \in$ $R_{k}(F)$ and $p=\left(p_{1}, \ldots, p_{k}\right)$ is a polynomial map $p(h(a))=h(p(a)) \in$ $p(\widehat{a})(F)=p(\widehat{a}(F))$, so that $h(a) \in R_{k}(\widehat{a} X(A)(F))$. To see the converse inclusion let $z \in R_{k}(\widehat{a}(F))$. Observe that $R_{k}(\widehat{a}(F)) \subset R_{k}(\sigma(a))=$ $\sigma(a)$ because the joint spectrum of a system of generators is always polynomially convex, a fortiori $k$-rationally convex for every $k \geq 1$. Then $z=h(a)$ for some $h \in X(A)$, and $p(z) \in p(\widehat{a}(F))$ for all $p$, that is $h(p(a)) \in p(\widehat{a})(F)$ for all $p$. Now, using the fact that the set of all $p(a)$ is dense in $A^{k}$ and applying $1.3(\mathrm{vi})$ we conclude that $h \in R_{k}(F)$. Thus, $R_{k}(\widehat{a}(F)) \subset \widehat{a}\left(R_{k}(F)\right)$.

5.6. TheOREM. If $A$ is a complex commutative Banach algebra with identity and $d(A)$ is the dimension of its spectrum $X(A)$ then

$$
d(A) \leq \gamma(A)+r(A) \leq 2 \gamma(A) .
$$

Proof. By 3.2 (ii) $r(A) \leq \gamma(A)$ and it suffices to prove the first inequality. Let $n=\gamma(A)$ and $k=r(A)$. By [14, Ch. VIII] it suffices to prove that $H^{n+i}(F)=0$ for all closed subsets $F$ of $X(A)$ and $i \geq k$. For $i \geq n+1, H^{n+i}(F)=0$ because $F$ is homeomorphic to some compact subset of $\mathbb{C}^{n}$. For $k \leq i \leq n$ we prove the assertion by a reverse induction.

Consider a (fixed) homeomorphism $\widehat{a}: X(A) \rightarrow \sigma(a) \subset \mathbb{C}^{n}$, and let $T=\sigma(a)$.

(+) Suppose that $H^{n+j}(F)=0$ for $j \geq i>k$ and for all closed subsets $F$ of $X(A)$. 
(a) Consider first a compact set of the form

$$
P=\bigcup_{j=1}^{k}\left\{z \in T:\left|p_{j}(z)\right| \geq \delta_{j}\right\}
$$

for some polynomials $p_{1}, \ldots, p_{k}$, and positive numbers $\delta_{1}, \ldots, \delta_{k}$. Let $R_{j}=\left\{z \in T:\left|p_{j}(z)\right| \geq \delta_{j}\right\}$. Then

$$
H^{n+i-1}(P)=H^{n+i-1}\left(\bigcup_{j=1}^{k} R_{j}\right)=0 \quad \text { by } 6.1 \text {. }
$$

(b) Let $\mathscr{P}$ be the collection of all $P$ like in (a). If $J=\bigcap_{l=1}^{m} P_{l}\left(P_{l} \in\right.$ $\mathscr{P})$ then $H^{n+i-1}(J)=0$. We prove this assertion by induction in $m$. For $m=1$, it has been proved at (a). Suppose that it holds for $1, \ldots, m$ and let $P_{1}, \ldots, P_{m+1} \in \mathscr{P}, E=\bigcap_{l=1}^{m} P_{l}, F=P_{m+1}$ and $r=n+i-1$. Consider the portion of the Mayer-Vietoris exact sequence

$$
\cdots \rightarrow H^{r}(E) \oplus H^{r}(F) \rightarrow H^{r}(E \cap F) \rightarrow H^{r+1}(E \cup F) \rightarrow \cdots .
$$

Then $H^{r}(E)=0$ by inductive hypothesis, $H^{r}(F)=0$ by (a) and $H^{r+1}(E \cup F)=0$ by $(+)$ (that is, by our first inductive hypothesis), because $E \cup F$ is homeomorphic to a closed subset of $X(A)$. Thus

$$
H^{n+i-1}\left(\bigcap_{l=1}^{m+1} P\right)=H^{r}(E \cap F)=0,
$$

which proves the assertion of (b).

Finally, we consider a closed subset $F$ of $X(A)$ and its image $F^{\prime} \subset$ $\mathbb{C}^{n}$ by $\widehat{a}: X(A) \rightarrow T$.

$F^{\prime}$ is $k$-rationally convex by 5.5 , so that $F^{\prime}=\bigcap Q_{\alpha}$ for some (possibly infinite collection of) $Q_{\alpha} \in P$. For each $Q_{\alpha}$ it holds $H^{n+i-1}\left(Q_{\alpha}\right)=0$ by (b). Thus, by the continuity of Čech cohomology we get $H^{n+i-1}(F)$ $=H^{n+i-1}\left(F^{\prime}\right)=0$, which finishes the proof.

5.7. Corollary. If $d(A)=2 n$ and $\gamma(A)=n$ then $r(A)=n$.

5.8. Examples. (i) Let $X \subset \mathbb{C}^{n}$ be the polydisc $\mathbb{D}^{n}$ or the ball $B_{n}$ or any polynomially convex compact subset of $C^{n}$ with interior. Then $r(A(X))=n$.

(ii) Let $A\left(\mathbb{D}^{\infty}\right)$ be the closure in $C\left(\mathbb{D}^{\infty}\right)$ of all polynomials in a finite number of variables.

Observe that every $A\left(\mathbb{D}^{n}\right)$ is a quotient of $A\left(\mathbb{D}^{\infty}\right)$. 
It is easy to prove, in general, that $r(A / I) \leq r(A)$ for all ideals $I$ of $A$.

Then $r\left(A\left(\mathbb{D}^{\infty}\right)\right) \geq r\left(A\left(\mathbb{D}^{n}\right)\right)=n$ for all $n$, by 5.7 .

Thus, $r\left(A\left(D^{\infty}\right)\right)=\infty$.

(iii) This is an example of a $k$-rationally convex compact subset of $\mathbb{C}^{n}$ which is not the union of two 1-rationally convex sets. Consider

$$
\begin{aligned}
& K_{1}=\left\{z \in D^{4}:\left|z_{1}\right| \geq 1 / 2 \text { or }\left|z_{2}\right| \geq 1 / 2\right\}, \\
& K_{2}=\left\{z \in D^{4}:\left|z_{3}\right| \geq 1 / 2 \text { or }\left|z_{4}\right| \geq 1 / 2\right\} .
\end{aligned}
$$

It is clear that $K_{1}, K_{2}$ and $K_{1} \cap K_{2}$ are 2-rationally convex sets. We consider the exact sequence

$$
\begin{aligned}
\cdots & \rightarrow H^{6}\left(K_{1}\right) \oplus H^{6}\left(K_{2}\right) \rightarrow H^{6}\left(K_{1} \cap K_{2}\right) \rightarrow H^{7}\left(K_{1} \cup K_{2}\right) \\
& \rightarrow H^{7}\left(K_{1}\right) \oplus H^{7}\left(K_{2}\right) \cdots .
\end{aligned}
$$

Now $H^{6}\left(K_{1}\right)=H^{6}\left(K_{2}\right)=0$ because $K_{1}$ and $K_{2}$ are unions of two 1 -rationally convex sets. By the same reason, $H^{7}\left(K_{1}\right)=H^{7}\left(K_{2}\right)=0$. Thus, $H^{6}\left(K_{1} \cap K_{2}\right) \cong H^{7}\left(K_{1} \cup K_{2}\right) \neq 0$, because $K_{1} \cup K_{2}$ separates $\mathbb{C}^{4}$, so that by $5.1 K_{1} \cup K_{2}$ is not the union of two 1-rationally convex sets.

6. A result of Forelli. Our generalized rational hulls give a notion of separation associated to $n$-tuples of elements of $A$. In this section we consider $n$-tuples of elements of an ideal $I$ of $A$. In [11] Forelli proves that, if $A$ is the disc algebra, $I$ is an ideal of $A$ and $F$ is a compact subset of $\mathbb{D}$ such that $F \cap \operatorname{hull}(I)=\varnothing$ (where hull $(I)=$ $\{z \in \mathbb{D}: f(z)=0 \forall f \in I\})$ then there exists $f \in I$ such that $f \mid F$ does not vanish. Here we generalize Forelli's theorem in the following sense:

6.1. TheOREM. Let $A$ be n-generated, $F$ a closed subset of $X(A)$ such that $F \cap \operatorname{hull}(I)=\varnothing$. Then there exist $a=\left(a_{1}, \ldots, a_{n}\right) \in I^{n}$ with $0 \notin \widehat{a}(F)$.

Proof. First we suppose that $A$ is uniform. Then $A$ is $A(X)$ for some polynomially convex compact $X \subset \mathbb{C}^{n}$. Now, if $V$ is an open neighborhood of hull( $I)$ such that $V \cap F=\varnothing$, then by the polynomial convexity of hull $(I)$ and a result of Gunning and Rossi [13, p. 218] there exist $n$ polynomials $p_{1}, \ldots, p_{n}$ in $\mathbb{C}\left[t_{1}, \ldots, t_{n}\right]$ such that

$$
V \supset\left\{z \in X:\left|p_{1}(z)\right|<1, \ldots,\left|p_{n}(z)\right|<1\right\} \supset \operatorname{hull}(I) .
$$

Define $C_{k}=\left\{z \in X:\left|p_{k}(z)\right| \geq 1\right\}$. Then $\operatorname{hull}(I) \cap C_{k}=\varnothing, C_{k}$ is rationally convex $(k=1, \ldots, n)$ and $F \subset \bigcup_{k=1}^{n} C_{k}$. Fix $k$. By compactness, there exists $g=\left(g_{1}, \ldots, g_{m}\right) \in I^{m}$ such that $0 \notin g\left(C_{k}\right)$. 
The spectrum of $R\left(C_{k}\right)$ (the closed subalgebra generated by the rational functions with poles off $\left.C_{k}\right)$ is $C_{k}$, is rationally convex. Thus, $0 \notin g\left(C_{k}\right)$ means that $g \in U_{m}\left(R\left(C_{k}\right)\right)$ and, by the definition of $R\left(C_{k}\right)$, there exist polynomials $r_{j}, s_{j}(j=1, \ldots, m)$ such that $s_{j}$ does not vanish on $C_{k}$ and $h=\sum_{j=1}^{m} r_{j} g_{j} / s_{j}$ does not vanish on $C_{k}$. Thus $f_{k}=\left(\prod_{j=1}^{m} s_{j}\right) h \in I$ and $f_{k}$ does not vanish on $C_{k}$. Now, $k$ being arbitrary, we get $f=\left(f_{1}, \ldots, f_{n}\right) \in I^{n}$ such that $0 \notin f\left(\bigcup_{k=1}^{n} C_{k}\right)$ and, a fortiori, $0 \notin f(F)$, as claimed.

For $A$ not necessarily uniform, we only need to observe that, in the proof above, we work with elements in $\widehat{A}$ so the result holds in general.

6.2. Corollary. Under the same hypothesis if $V \subset X(A)$ is a neighborhood of hull $(I)$ then there exists an n-generated ideal $J \subset I$ such that hull $(I) \subset \operatorname{hull}(J) \subset V$.

Proof. It suffices to take $F=X(A) \backslash V$ and $J=\left\langle f_{1}, \ldots, f_{n}\right\rangle$.

7. Some results on $H^{\infty}$. The famous "corona theorem" of Carleson [3] says that if $f_{1}, \ldots, f_{n}$ are bounded holomorphic functions on the open disc $\Delta$ (in symbols, $f_{1}, \ldots, f_{n} \in H^{\infty}$ ) such that $\left|f_{1}\right|+\cdots+\left|f_{n}\right| \geq \delta$ for some $\delta>0$ then there exist $g_{1}, \ldots, g_{n} \in H^{\infty}$ such that $\sum_{k=1}^{n} f_{k} g_{k}=$ 1 ; if we suppose $\Delta$ imbedded in $X\left(H^{\infty}\right)$, Carleson's result says that $\Delta$ is dense. The rationality of $H^{\infty}$ is closely related to the corona theorem. More precisely, if we knew that $r\left(H^{\infty}\right) \leq m$ then it would suffice to prove Carleson's theorem for $m$-tuples. In fact, if there exists $\varphi \in H(H) \backslash \bar{\Delta}$ (here $\bar{\Delta}$ is the closure of $\Delta$ in $X\left(H^{\infty}\right)$ ), then there is an $f=\left(f_{1}, \ldots, f_{r}\right) \in\left(H^{\infty}\right)^{r}$ with $r=r\left(H^{\infty}\right)$ such that $\widehat{f}(\varphi)=0$ and $\widehat{f}(x) \neq 0$ for all $x \in \bar{\Delta}$. We may assume that $f \in\left(H^{\infty}\right)^{m}$, filling the last coordinates with zeros if it is necessary. Thus, by compactness of $\bar{\Delta},|f(z)|=\left|f_{1}(x)\right|+\cdots+\left|f_{m}(z)\right| \geq \delta>0$ for some $\delta$ and all $z \in F$, so $f$ verifies the corona hypothesis and then, by our assumption, $f \in U_{m}\left(H^{\infty}\right)$, which contradicts the existence of $\varphi$.

In particular, if we knew that $r\left(H^{\infty}\right)=1$ this would imply trivially Carleson's theorem. We collect in this section a few results about the hull $R_{n}$ of $H^{\infty}$.

We recall that $\left\{z_{n}\right\} \subset \Delta$ is an interpolating sequence if for every bounded sequence $\left\{b_{n}\right\}$ there exists $f \in H^{\infty}$ such that $f\left(z_{n}\right)=b_{n}$ $(n \geq 1)$. By identifying the points of $\Delta$ with some characters of $H^{\infty}$, we define $G=\left\{x \in X\left(H^{\infty}\right): x\right.$ is a cluster point of an interpolating sequence $\left.\left\{z_{n}\right\}\right\}$. 
Obviously $G \supset \Delta$ and it can be shown that $G \cap S_{0}\left(H^{\infty}\right)=\varnothing$ but $G \cup S_{0}\left(H^{\infty}\right)$ is strictly contained in $X\left(H^{\infty}\right)$.

7.1. THEOREM. Let $F$ be a compact subset of $X\left(H^{\infty}\right)$.

(1) If $F \subset S_{0}\left(H^{\infty}\right)$ then $R_{1}(F)=F$.

(2) If $F \subset X\left(H^{\infty}\right)$ then

$$
R_{2}(F) \backslash F \subset X\left(H^{\infty}\right) \backslash G .
$$

Proof. (1) Let $x \in X\left(H^{\infty}\right) \backslash S_{0}\left(H^{\infty}\right)$. By a theorem of Newman $[12$, p. 194] there is a Blaschke product $B$ such that $B(x)=0$ and $|B| F \mid \equiv 1$. Then $x \notin R_{1}(F)$. This proves that $R_{1}(F) \subset S_{0}\left(H^{\infty}\right)$. Let $x \in S_{0}\left(H^{\infty}\right) \backslash F$. Recall that $X\left(L^{\infty}\right) \approx S_{0}\left(H^{\infty}\right)$ and $\widehat{L}^{\infty}=C\left(X\left(L^{\infty}\right)\right)$. Then, there is $u \in L^{\infty}$ such that $u(F)=1,|u| \equiv 1$ and $u(x)=-1$. By a theorem of Douglas and Rudin [12, p. 192] there exist Blaschke products $B_{1}$ and $B_{2}$ such that $\left\|B_{1} / B_{2}-u\right\|_{S_{0}\left(H^{\infty}\right)}<\varepsilon<1$, if $\varepsilon>0$. Let $h=B_{1}-B_{2}$. Then, if $z \in F$

$$
|h(z)|=\left|B_{1}(z)-u(z) B_{2}(z)\right|<\varepsilon\left|B_{2}(z)\right|=\varepsilon
$$

and

$$
\begin{aligned}
|h(x)| & =\left|B_{1}(x)+B_{2}(x)-2 B_{2}(x)\right| \\
& \geq|| 2 B_{2}(x)|-| B_{1}(x)+B_{2}(x) \| \\
& =2-\left|B_{1}(x)+B_{2}(x)\right| \\
& =2-\left|B_{1}(x)-u(x) B_{2}(x)\right|>2-\varepsilon .
\end{aligned}
$$

This proves that $|h(x)|>\|h\|_{F}$, a fortiori $x \notin R_{1}(F)$.

(2) Let $x \in G \backslash F$. Then there is an interpolating sequence $\left\{z_{n}\right\}$ such $x \in L=$ closure of $\left\{z_{n}: n \in \mathbb{N}\right\}$. Let $Q: H^{\infty} \rightarrow l^{\infty}$ be defined by $Q(f)=\left\{f\left(z_{n}\right)\right\}_{n \in \mathbb{N}}$. Then $Q$ is onto and $\widehat{Q}: \widehat{H}^{\infty} \rightarrow \widehat{l}^{\infty}=C\left(X\left(l^{\infty}\right)\right)=$ $C(L)$ is onto, too, because $Q^{*}: X\left(l^{\infty}\right) \rightarrow L$ is a homeomorphism.

Let $g \in C(L)$ such that $Z_{g} \cap(F \cap L)=\varnothing$. Then there is $f \in H^{\infty}$ such that $f \mid L=g$. Thus, if $B$ is a Blaschke product whose zeros $a_{n}(n \in \mathbb{N})$, then $(B, f)$ separates $x$ from $F$, for $(B, f)(x)=0$ and if $z \in F$ and $B(z)=0$ by [15, pp. 379] $z \in L$ so that $f(z)=g(z)$ $\neq 0$.

8. Some open problems. There are several questions about the subjects considered in this paper that we have not been able to answer. We collect in this section those that we consider the most relevant.

8.1. $H^{\infty}$. As usual in uniform algebra theory, this algebra is a source of many interesting questions. We do not know neither the 
dimension of its spectrum nor its rationalilty $r\left(H^{\infty}\right)$. It is possible that $r\left(H^{\infty}\right)=+\infty$. We also ignore its Bass' stable rank and its topological stable rank.

8.2. $r(A)$ vs. $\operatorname{tsr}(A)$. We suspect that $r(a) \leq \operatorname{tsr}(A)-1$, but we do not have a proof even for the case $\operatorname{tsr}(A)=1$.

8.3. $\operatorname{tsr}(A)$ vs. $\operatorname{tsr}(C(X(A)))$. These two invariants should be related, but we only have partial answers (see $\S 4)$.

8.4. Is it true that $S_{n-1}(A)=X(A)$ if $\operatorname{tsr}(A) \leq n$ ?

\section{REFERENCES}

[1] R. Basener, A generalized Shilov boundary and analytic structure, Proc. Amer. Math. Soc., 47 (1975), 98-104.

[2] H. Bass, K-theory and stable algebra, Publ. Math. $\mathrm{N}^{\circ} 22$ (1964), 489-544.

[3] L. Carleson, Interpolation by bounded analytic functions and the Corona problem, Ann. of Math., 76 (1962), 547-559.

[4] G. Corach and F. D. Suárez, Thin spectra and stable range conditions, J. Funct. Anal., 81 (1988), 432-442.

[5] G. Csordas and H. Reiter, Separating function algebras, Nagoya Math. J., 47 (1972), 101-109.

[6] R. Curto, Applications of several complex variables to multiparameter spectral theory, in Survey of Recent Results in Operator Theory, vol. II, J. B. Conway and B. B. Morrell eds., Longman's, London, 1988, pp. 25-90.

[7] T. Duchamp and E. L. Stout, Maximum modulus sets, Ann. Inst. Fourier (Grenoble), 31 (1981), 37-69.

[8] L. Q. Eifler, Nonseparating function algebras, Bull. Amer. Math. Soc., 78 (1972), 604-605.

[9] L. Q. Eifler, Inverting sets for function algebras, Proc. Amer. Math. Soc., 37 (1973), 92-96.

[10] J. Eschmeier, Analytic spectral mapping theorems for joint spectra, Operator Theory: Advances and Applications, Vol. 24, pp. 167-181, Birkhäuser, Basel, 1987.

[11] F. Forelli, A note on ideals in the disc algebra, Proc. Amer. Math. Soc., 84 (1982), 389-392.

[12] J. Garnett, Bounded Analytic Functions, Academic Press, New York, 1981.

[13] R. Gunning and H. Rossi, Analytic Functions of Several Complex Variables, Prentice Hall, Englewood Cliffs, N. J., 1965.

[14] W. Hurewicz and H. Wallman, Dimension Theory, Princeton University Press, Princeton, 1941.

[15] M. Rieffel, Dimension and stable rank in the $K$-theory of $C^{*}$-algebras, Proc. London Math. Soc., (3) 46 (1983), 301-333.

[16] W. Rudin, Function Theory in Polydiscs, Benjamin, New York-Amsterdam, 1969.

[17] N. Sibony, Multi-dimensional analytic structure in the spectrum of uniform algebras, Lecture Notes in Math., Vol. 512, Springer-Verlag, Berlin, pp. 139-169.

[18] Z. Slodkowski, Analytic perturbation of the Taylor spectrum, Trans. Amer. Math. Soc., 297 (1986), 319-336. 
[19] E. L. Stout, The Theory of Uniform Alegbras, Bogdon \& Quigley, Tarrytownon-Hudson, 1971.

[20] J. L. Taylor, Topological invariants of the maximal ideal space of a Banach algebra, Adv. in Math., 19 (1976), 149-206.

[21] T. V. Tonev, Minimal boundaries of commutative Banach algebras, containing the Shilov boundary, in Complex analysis and applications Varna' 85, Sofia (to appear).

[22] L. N. Vaserstein, Stable range of rings and dimensionality of topological spaces, Funct. Anal. Appl., 5 (1971), 102-110.

[23] F.-H. Vasilescu, A multidimensional spectral theory in $C^{*}$-algebras, Banach Center Publ., vol. 8 (Spectral theory) (1982), 471-491.

[24] D. Wilken, Approximate Normality and Function Algebras on the Interval and Circle, in Function algebras (ed. F. T. Birtel), Scott, Foreman and Co., Chicago, 1966, pp. 98-111.

[25] W. Zelazko, An axiomatic approach to joint spectra, I., Studia Math., 64 (1979), 250-261.

Received June 15, 1987 and in revised form September 10, 1988.

Consejo Nacional de Investigaciones Cientificas y Tecnicas

VIAMONTE 1636

$1^{\text {er }}$ Cuerpo, $1^{\text {er }}$ Piso

1055 Buenos Aires, Argentina 



\section{PACIFIC JOURNAL OF MATHEMATICS EDITORS}

\author{
V. S. VARADARAJAN \\ (Managing Editor) \\ University of California \\ Los Angeles, CA 90024-1555-05 \\ Herbert Clemens \\ University of Utah \\ Salt Lake City, UT 84112 \\ THOMAS ENRIGHT \\ University of California, San Diego \\ La Jolla, CA 92093
}

R. FINN

Stanford University

Stanford, CA 94305

HeRMANN FlaschKa

University of Arizona

Tucson, AZ 85721

VAUGHaN F. R. Jones

University of California

Berkeley, CA 94720

STEVEN KeRCKHOFF

Stanford University

Stanford, CA 94305

\author{
ROBION KIRBY \\ University of California \\ Berkeley, CA 94720 \\ C. C. MOORE \\ University of California \\ Berkeley, CA 94720 \\ HAROLD STARK \\ University of California, San Diego \\ La Jolla, CA 92093
}

\section{ASSOCIATE EDITORS}
R. ARENS
E. F. BECKENBACH
B. H. NEUMANN
F. WOLF
K. YoshidA (1906-1982)

\section{SUPPORTING INSTITUTIONS}

UNIVERSITY OF ARIZONA

UNIVERSITY OF BRITISH COLUMBIA

CALIFORNIA INSTITUTE OF TECHNOLOGY

UNIVERSITY OF CALIFORNIA

MONTANA STATE UNIVERSITY

UNIVERSITY OF NEVADA, RENO

NEW MEXICO STATE UNIVERSITY

OREGON STATE UNIVERSITY
UNIVERSITY OF OREGON

UNIVERSITY OF SOUTHERN CALIFORNIA

STANFORD UNIVERSITY

UNIVERSITY OF HAWAII

UNIVERSITY OF TOKYO

UNIVERSITY OF UTAH

WASHINGTON STATE UNIVERSITY

UNIVERSITY OF WASHINGTON 


\section{Pacific Journal of Mathematics}

Vol. 140, No. $1 \quad$ September, 1989

Michel Brestovski, Algebraic independence of solutions of differential

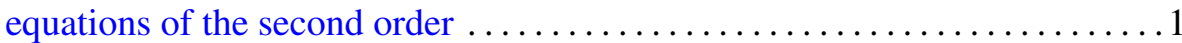

Bohumil Cenkl, Cohomology operations from higher products in the de

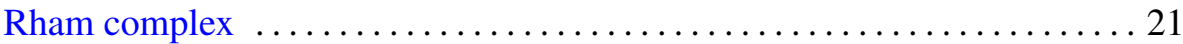

Gustavo Corach and Daniel Suarez, Generalized rational convexity in

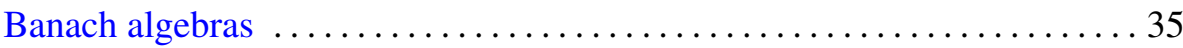

Keresztély Corrádi and Sándor Szabó, A new proof of Rédei’s theorem . . . 53

Steven R. Costenoble and Stefan Waner, Equivariant orientations and

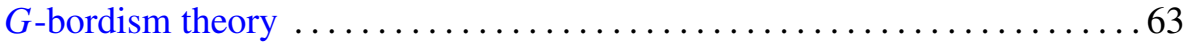

Angel Granja, Apéry basis and polar invariants of plane curve

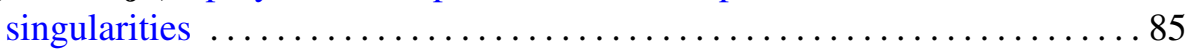

Young Soo Jo, Isometries of tridiagonal algebras .................. 97

Ronald Leslie Lipsman, Harmonic analysis on exponential solvable homogeneous spaces: the algebraic or symmetric cases $\ldots \ldots \ldots \ldots \ldots 117$

Erich Miersemann, On the behaviour of capillaries at a corner 149

Marian Nowak, On the finest Lebesgue topology on the space of essentially

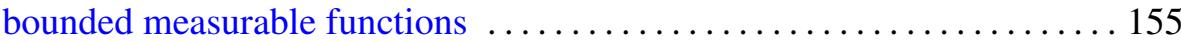

Pascal J. Thomas, Hardy interpolating sequences of hyperplanes ........ 163

H. Bevan Thompson, Differentiability properties of subfunctions for second order ordinary differential equations 